\title{
LE TYPE ET L'AUTHENTICITÉ DU TEXTE ET LEUR INFLUENCE SUR LA COMPRÉHENSION ${ }^{1}$
}

\section{INTRODUCTION}

L'objet de cette étude est d'analyser le rôle du texte dans la compréhension écrite, notamment sous deux de ses aspects: le type et l'authenticité. Le but est surtout pratique: en apprendre plus sur le rôle du texte pour pouvoir ensuite mieux enseigner et évaluer la compréhension écrite.

Dans la pratique pédagogique il arrive souvent que les exercices de compréhension écrite proposés aux élèves ${ }^{2}$ ne les différencient pas assez, ce qui soulève certaines questions sur ce qui est plus facile ou plus difficile pour les élèves. Pour l'élaboration des exercices l'utilisation de textes adaptés de façon intuitive est une pratique courante et il convient de se demander si les textes adaptés de cette façon facilitent vraiment la compréhension.

L'étude est basée sur la typologie de J.-M. Adam que nous avons employée d'abord pour l'analyse du corpus et ensuite pour l'élaboration des exercices employés lors de l'expérimentation en classe. Au critère du type de texte nous avons ajouté celui de l'authenticité: tous les exercices étaient proposés en deux variantes (authentique et adaptée).

Le corpus dans lequel nous avons d'abord analysé les textes selon les critères du type et de l'authenticité était constitué de trois manuels de FLE:

- Le nouveau sans frontières (1988),

- Panorama (1996),

- Campus (2002).

Les manuels ont été choisis en fonction de deux critères: leur diffusion en Slovénie $^{3}$ et la présence du même auteur (J. Girardet) dans les trois manuels.

1 L'article résume la thèse de doctorat Vpliv tipa in avtentičnosti besedila na njegovo razumevanje, soutenue le 24 juin 2004 à la Faculté des lettres de Ljubljana devant le jury prof. V. Pogačnik (président du jury), prof. Mojca Schlamberger Brezar (directrice de thèse), prof. Ana Marija Muster (membre).

2 Ici nous pensons aux exercices du baccalauréat et du concours national de français pour les lycéens slovènes.

3 Le nouveau sans frontières et Panorama sont (étaient) les manuels les plus employés en Slovénie. Nous avons ajouté une méthode plus récente, Campus, à cause du même auteur. 


\section{LES TYPES DE TEXTES}

Bien que critiquée par certains linguistes (Roulet, 1991: 120, Reboul et Moeschler, 1998: 100), la typologie élaborée par J.-M. Adam semble être la plus utilisée dans le monde du FLE. Le choix de cette typologie pour l'analyse du corpus et l'élaboration des exercices s'appuie aussi sur le fait qu'une typologie similaire est présentée chez nous en classe de langue maternelle (Bešter et al., 1999: 92) et devrait donc être familière aux élèves slovènes.

La typologie d'Adam est une typologie qui traite de l'organisation cognitive, sousjacente de certaines séquences. L'auteur se réfère à des schémas textuels prototypiques dont «la maitrise semble avoir des conséquences sur le stockage des informations traitées en cours de compréhension et sur la recherche des blocs d'information par stratégies d'information» (Adam, 1997: 7). ${ }^{4}$

Après s'être appuyé, entre autres, sur les travaux de Kintsch et de van Dijk (p. ex. Toward a Model of Text Comprehension and Production, 1978) J.-M. Adam a affiné sa typologie en se basant sur les idées de M. Bakhtine. Il s'est inspiré surtout de deux de ses idées: celles qui déterminent la compétence linguistique au-delà de la phrase, notamment de l'idée des «types relativement stables d'énoncés» et de la «syntaxe des grandes masses verbales» (Adam, 1997: 11).

Selon Adam (1999: 82) on ne peut pas parler de types de textes car «l'unité texte est trop complexe et trop hétérogène pour présenter des régularités linguistiquement observables et codifiables». Pour cela, il introduit la notion de séquence qu'il définit comme:

- un réseau relationnel hiérarchique: grandeur décomposable en parties reliées entre elles,

- une entité relativement autonome, dotée d'une organisation interne qui lui est propre et donc en relation de dépendance/indépendance avec l'ensemble plus vaste dont elle fait partie (1997: 28).

Chaque séquence est constituée de paquets de propositions (les macro-propositions), elles mêmes constituées de propositions:

[ \# TEXTE \# [ Séquence(s) [ macro-proposition(s) [ proposition(s)]]] ]

(Adam, 1997: 30)

Adam introduit pourtant la notion de dominante textuelle qui s'avère très utile dans notre cas: même si un texte comporte des séquences appartenant à des types

4 Adam consacre une grande partie de son travail aux notions de super- et macrostructures que nous n'approfondirons pas, vu que notre recherche est surtout orientée vers la pratique.

5 Adam utilise le signe \# pour la délimitation des frontières du texte, marques de début et de fin d'une communication (1997: 30). 
différents, il est presque toujours possible d'affirmer qu'il s'agit plutôt d'une narration, d'une description,... donc, de texte à dominante narrative, descriptive, etc.

Nous avons dans la plupart des cas gardé l'expression le type de texte (parfois le texte à dominante...): dans notre corpus on trouve surtout des textes composés d'une seule séquence ou de plusieurs séquences du même type, donc des textes plutôt homogènes.

Au fil des années, Adam a changé et affiné sa typologie ${ }^{6}$. Dans un de ses premiers articles sur ce thème, Quels types de textes (1985) il définit les types de texte narratif, descriptif, explicatif, argumentatif, injonctif, prédictif, conversationnel et rhétorique. Peu à peu, il a abandonné les types injonctif, prédictif et rhétorique pour conserver cinq types de base: la narration, la description, l'argumentation, l'explication et le dialogue (1991: 14-15, 1997).

\subsection{La narration 7}

Pour pouvoir parler de narration, le texte doit satisfaire à plusieurs critères:

- la succession temporelle d'actions,

- l'unité thématique (au moins un acteur-sujet): sans implication d'intérêt humain il n'y a pas de récit,

- des prédicats transformés: qu'il soit dit ce qu'il advient à l'instant $\langle\mathrm{t}+\mathrm{n}\rangle$ des prédicats qui caractérisent le sujet d'état [S] à l'instant $\langle\mathrm{t}\rangle$,

- un processus: unité d'une même action,

- la causalité-consécution narrative d'une mise en intrigue,

- une évaluation finale (explicite ou implicite)

(Adam, 1994: 46-58).

U. Eco insiste pourtant plus sur les événements inattendus ou inusuels: «... une narration est une description d'actions qui requiert pour chaque action décrite un agent, une intention de l'agent, un état ou monde possible, un changement avec sa cause et le propos qui le détermine; on pourrait ajouter à cela des états mentaux, des émotions, des circonstances; mais la description est importante (nous dirons : conversationnellement admissible) si les actions décrites sont difficiles et seulement si l'agent n'a pas un choix évident quant au cours des actions à entreprendre pour changer l'état qui ne correspond pas à ses propres désirs; les événements qui suivent cette décision doivent être inattendus, et certains d'entre eux doivent apparaître inusuels ou étranges» (Eco, 1985: 137).

6 Dans l'avant-propos de son livre Les textes, types et prototypes, il précise, que son propos “ $\mathrm{n}$ 'est pas de mettre un point final au(x) débat(s) en cours en proposant une typologie de plus ou LA typologie définitive» (1997: 9)

7 En français on trouve aussi d'autres expressions: le récit (employé aussi par Adam), l'histoire et les verbes raconter et relater. En slovène on emploie surtout les expressions pripoved et zgodba. 
L'exemple suivant, cité par Eco, n'est donc pas une narration:

Hier je suis sorti de chez moi pour aller prendre le train de $8 \mathrm{~h} 30$ qui arrive à Turin à 10 heures. J'ai pris un taxi qui m'a amené à la gare, là j'ai acheté un billet et je me suis rendu sur le bon quai; à 8 h 20 je suis monté dans le train qui est parti à

l'heure et qui m'a conduit à Turin.

parce qu'il lui manque justement un événement inattendu (le texte deviendrait néanmoins une narration, s'il y avait une bombe dans le train).

Le schéma de base d'une narration contient trois parties:

\begin{tabular}{|l|l|l|}
\hline $\begin{array}{l}\text { Situation initale } \\
\text { AVANT }\end{array}$ & $\begin{array}{l}\text { Transformation } \\
\text { PENDANT }\end{array}$ & $\begin{array}{l}\text { Situation finale } \\
\text { APRÈS }\end{array}$ \\
\hline
\end{tabular}

Adam propose un schéma plus élaboré, en sept parties, la première et la dernière n'étant pas obligatoires:

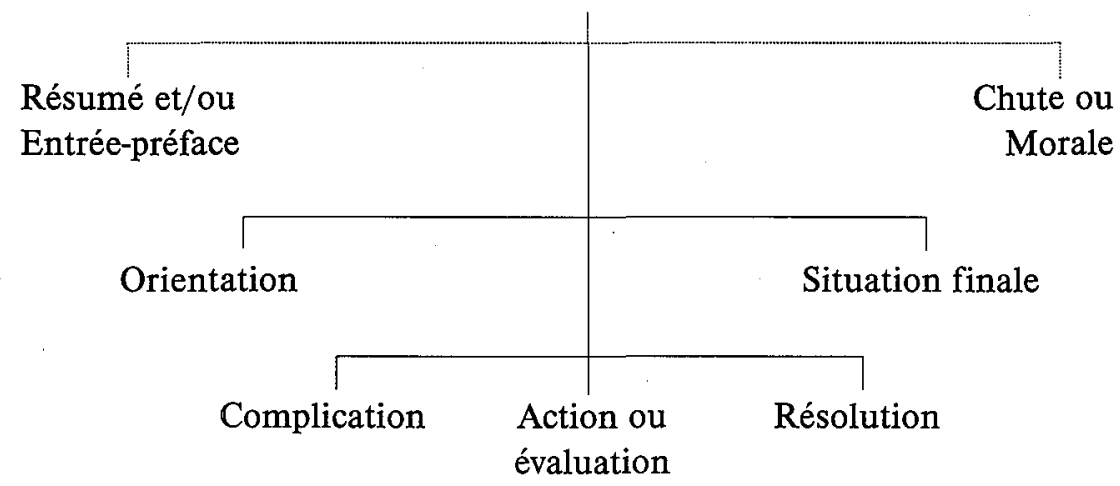

(d'après Adam, 1997: 94)

Un exemple de narration serait l'article du journal Libération, trouvé dans notre corpus:

\section{ABANDONNÉE DANS LES TOILETTES}

[1] Une fillette de trois ans a été découverte abandonnée dans des toilettes publiques à Monaco, par une passante qui l'avait entendue pleurer. [2] Elle était recroquevillée sur le sol, sous la pluie. [3] «Elle était affamée, elle a d'abord englouti des dragées, disant seulement 'encore', puis tout un paquet de biscuits", raconte un témoin. [4] «Nous lui avons demandé son nom, d'où elle venait, où étaient ses parents. [5] Tout ce qu'elle disait, c'était 'voiture, voiture'»... [6] Après 24 heures, elle a fini par prononcer son prénom.

Libération, 27/04/88

Le nouveau sans frontières, cahier d'exercices: 121 


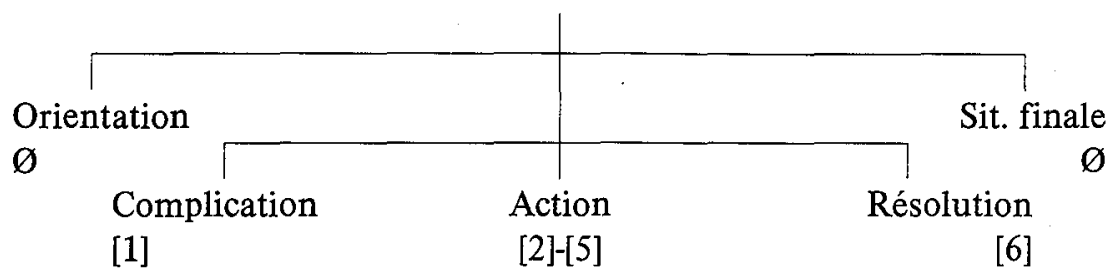

\subsection{La description}

La description «se construit autour d'une unité thématique (objet, lieu ou individu) dont sont pris en considération l'aspect général, les parties constitutives ou la situation dans le temps et l'espace» (Jeandillou, 1996: 149). Adam (1997: 85-93) distingue quatre parties de description:

- l'ancrage: peut se trouver au début (dans ce cas on l'appelle le thème-titre) ou à la fin de la description (le résumé); cette partie garantit l'unité sémantique de la séquence,

- l'aspectualisation est la base de la description: l'auteur du texte présente les différents aspects du thème - ses propriétés (dimensions, couleurs, forme,...) ou ses parties,

- la mise en relation rapproche le référent d'un ou de plusieurs autres référents, soit par une mise en situation (dans l'espace ou le temps), soit par une assimilation (par comparaison ou métaphore),

- la thématisation permet de continuer la description en introduisant de nouveau l'aspectualisation et la mise en relation.

Le schéma de la description est le suivant:

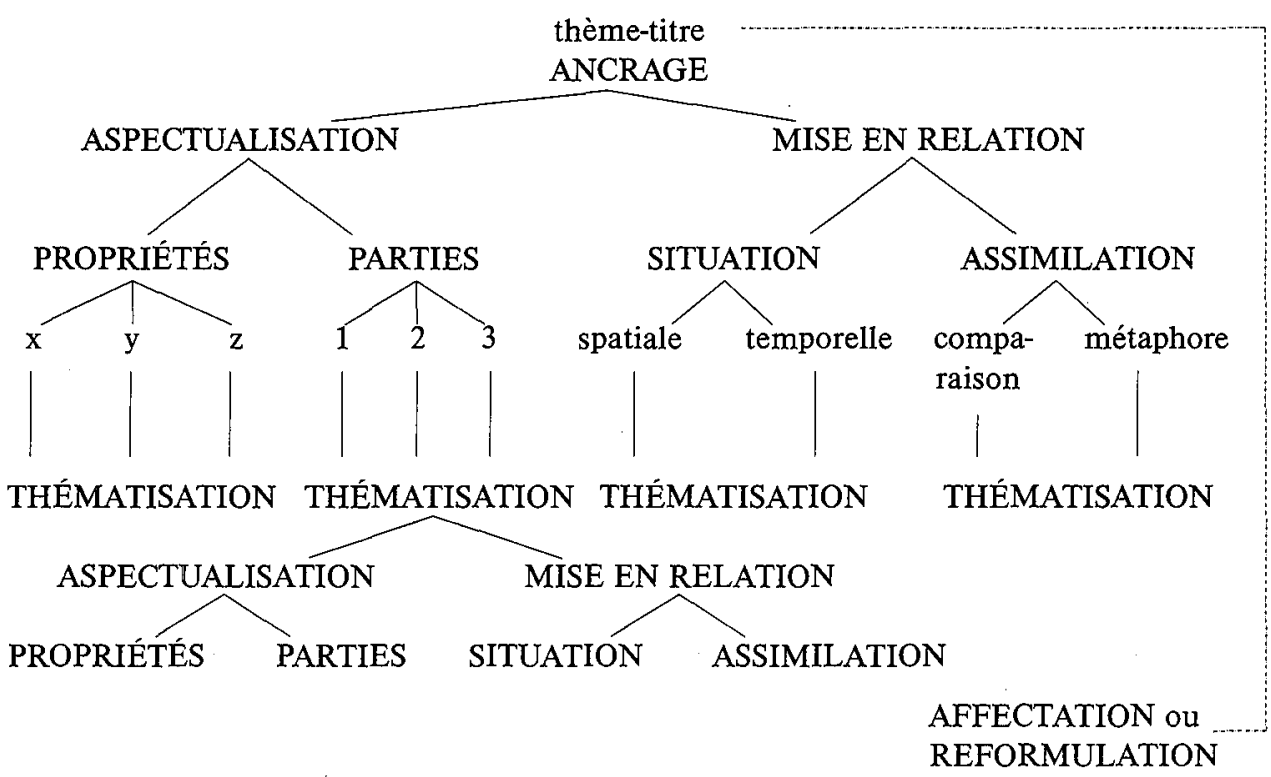

(Adam, 1997: 84, Jeandillou, 1996: 152) 
La plupart des descriptions s'inscrivent dans la partie gauche du schéma (aspectualisation: parties et propriétés).

Adam et Petitjean (1989: 157) mentionnent aussi un type limite de descriptions: ce sont les descriptions où le thème-titre déclenche une énumération dont l'ordre importe peu. Ils appellent ce type de descriptions la liste. C'est le type de description qui apparaît le plus souvent dans notre corpus.

Les textes, appelés d'habitude injonctifs ${ }^{8}$, présentent un problème. Ce sont des textes que la plupart des typologies, surtout celles employées en didactique du $\mathrm{FLE}^{9}$, mettent dans une catégorie à part; et dans ses premiers articles, Adam l'a également fait.

Ces textes disent de faire et se présentent «comme un algorithme de transformation, c'est-à-dire une suite ordonnée d'opérations qui permettent de passer d'un état 1 d'un système à un état 2» (Vigner, 1990: 107). L'auteur du texte définit les actions qui doivent, par la suite, être réalisées par le lecteur:

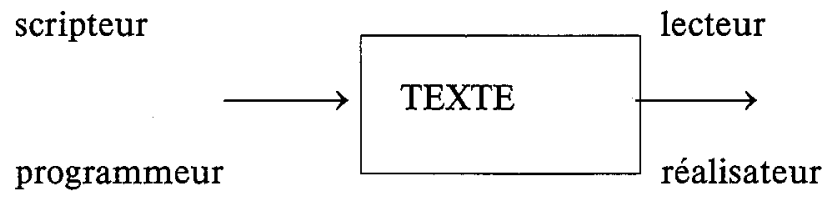

(Vigner: ibid.)

S'il s'agit d'une suite ordonnée d'opérations, on peut classer ces textes parmi les descriptions; Adam et Petitjean (1989: 152) les appellent les descriptions d'actions ${ }^{10}$. Voici le texte «injonctif» de notre corpus:

La recette des crêpes

$250 \mathrm{~g}$ de farine, 4 oeufs, $1 / 2$ litre de lait, 1 cuillère à soupe de sucre, 1 cuillère à café de sel, $50 \mathrm{~g}$ de beurre.

PRÉPARATION DE LA PATTE

Mélanger la farine avec les oeufs, le sel, le sucre et le lait

Ajouter le beurre

Attendre une ou deux heures

Mettre un peu de beurre dans une poêle

Mettre sur le feu

Verser un peu de pâte

8 Adam cite d'autres expressions désignant ce type de textes: textes régulateurs, procéduraux, programmateurs, instructionnels-prescriptifs, injonctifs-instructionnels, textes de conseil (Adam, 2001: 11).

9 Par exemple, la typologie présentée par Tagliante (1994).

10 Eco, lui, emploie la même expression pour désigner la narration. En slovène nous avons employé l'expression opis poteka. 
Attendre une minute et retourner la crêpe

(Le nouveau sans frontières: 89)

Il pourrait être analysé comme une description en deux parties: la première regroupant les parties du thème-titre (les ingrédients) et la deuxième incluant la description d'actions (mélanger, ajouter, ...).

\subsection{L'explication}

L'explication est en principe «une relation de communication entre deux agents, relativement à un objet: le locuteur $\mathrm{A}$ fait savoir ou fait comprendre à son interlocuteur B ce qu'est un certain objet (qui cause problème) en le décrivant, en l'analysant devant lui, en explicitant éléments ou aspects» (Garcia-Debanc, cité dans Pouliot, 1993: 122). Le texte explicatif répond à la question «Pourquoi?» ou «Comment?» qui peuvent être formulées de façons différentes, p.ex.: Mais au fond pourquoi? Qu'est-ce qui fait que?.

Le schéma de la séquence explicative selon Adam (1997: 132) a quatre parties:

\begin{tabular}{|l|l|l|l|}
\hline $\begin{array}{l}\text { Schématisation } \\
\text { initiale }\end{array}$ & $\begin{array}{l}\text { Problème } \\
\text { (question) }\end{array}$ & $\begin{array}{l}\text { Explication } \\
\text { (réponse) }\end{array}$ & $\begin{array}{l}\text { Conclusion - } \\
\text { évaluation }\end{array}$ \\
\hline & $\begin{array}{l}\text { POURQUOI X? } \\
\text { COMMENT X? }\end{array}$ & PARCE QUE... & \\
\hline
\end{tabular}

Nous avons inclus dans notre corpus le texte explicatif suivant:

Langues étrangères

[I] ILS ONT PEUR DE PARLER

POURQUOI?

Extraits d'interviews de touristes et d'étudiants français à Londres

[2] J'habite avec une copine française. [3] Elle parle très bien anglais. [4] Quand on est avec des amis anglais, je la laisse parler. [5] Je ne peux pas dire un mot.

Céline, 18 ans, étudiante

$\begin{array}{llll} & & & \\ \text { Sch. initiale } & \text { Problème } & \text { Réponse } & \text { Conclusion } \\ & \text { Pourquoi? } & & \\ {[2]} & {[1]} & {[3][4]} & {[5]}\end{array}$

Campus: 101 


\title{
2.4 L'argumentation
}

Un texte argumentatif «vise à intervenir sur les opinions, attitudes ou comportements d'un interlocuteur ou d'un auditoire en rendant crédible ou acceptable un énoncé (conclusion) appuyé, selon des modalités diverses, sur un(e) autre argument/donnée/raison» (Adam, 1997: 104).

Le schéma d'une séquence argumentative prototypique se présente sous la forme suivante:

thèse antérieure + données (prémisses) étayage des
inférences donc probablement conclusion (premisses) 1 inferences

(d'après Adam, 1997: 118)

\author{
SAUF SI \\ restriction \\ SEULEMENT \\ SI \\ spécification
}

Les textes argumentatifs sont très rares dans les manuels de FLE, et pour la plupart il s'agit de textes argumentatifs que Boissinot (1999: 41) appelle «dilués». Ce sont des textes qui «dans le meilleur des cas suggèrent un problème sans que les termes de celui-ci soient jamais clairement formulés. Il s'agit d'une écriture journalistique et éclatée (...). La cohérence de ces textes est faible, souvent assurée seulement par la récurrence de surface d'un thème ou d'un mot».

\subsection{Le dialogue}

Le type de texte dialogal est le plus controverse et le plus critiqué de tous les types figurant dans la typologie d'Adam. Roulet (1991: 120) admet que le dialogue est caractérisé par un type particulier de superstructure, mais ajoute qu'on ne peut pas le mettre sur le même plan que les autres types, puisque ceux-ci se situent précisément à un rang différent dans la hiérarchie discursive. La plupart des typologies oppose le dialogue au monologue ${ }^{11}$.

Adam (1997: 145) reconnaît que les critiques acceptent les quatres types précédents ${ }^{12}$, mais rejettent majoritairement le dialogue: «La tendance générale consiste à mettre le dialogue-conversation nettement à part soit en lui accordant une place prédominante, soit en l'ignorant».

\footnotetext{
11 Cette distinction apparaît aussi dans le manuel de slovène pour lycéens (Bešter et al., 1999: 92).

12

La narration, la description, l'explication et l'argumentation.
} 
Même si nous sommes d'accord avec le fait qu'on ne puisse pas mettre le dialogue sur le même plan que les autres types de textes, nous l'avons gardé pour l'analyse: la majorité des textes «pédagogiques» de notre corpus est écrite sous forme de dialogue et il serait très difficile de les analyser si on ne gardait pas le schéma de la séquence dialogale.

Le dialogue est une suite hiérarchisée de séquences appelées échanges: l'échange est la plus petite unité dialogale composant l'interaction. Chaque échange est composé des interventions, qui sont les plus grandes unités monologales composant l'échange. L'acte de langage est la plus petite unité monologale constituant l'intervention (d'après Moeschler, 1985: 81).

Le dialogue peut contenir des séquences phatiques d'ouverture et de clôture (p.ex. la salutation). Pour cerner les changements de séquences transactionnelles, Adam (1997: 155) propose le critère thématique.

Le schéma de la séquence dialogale simple (d'après Adam, 1997: 159)

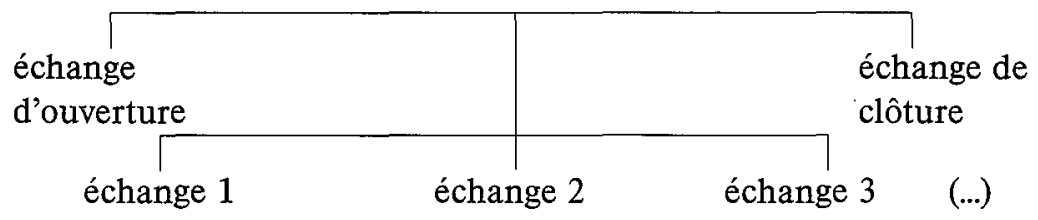

Adam (1997: 156) attire l'attention sur l'évaluation, qui représente, selon lui, un type d'intervention particulier: c'est l'échange par lequel un locuteur signale à l'autre qu'il a bien enregistré son intervention. Dans l'exemple

[A1]: Qu'est-ce que tu lis?

[B1]: Un bouquin de linguistique textuelle.

[A2]: Linguistique textuelle! / Ah bon! / Ben dis donc! / Très bien! / Ben merde!

l'évaluation est représentée par l'intervention [A2].

L'exemple de séquence dialogale suivant est tiré du Nouveau sans frontières:

\begin{tabular}{|l|l|l|}
\hline A1 & Pascal: & $\begin{array}{l}\text { [1] Pardon Madame, [1'] notre voiture est en panne. [2] Est-ce que je pourrais } \\
\text { téléphoner à Arles? }\end{array}$ \\
\hline B1 & Mireille: & [3] Bien sûr. [4] Mais Arles est à 40 kilomètres. [5] Le dépannage va coûter cher. \\
\hline A2 & Pascal: & [6] Alors, pourrions-nous camper dans votre propriété? \\
\hline B2 & Mireille: & [7] Normalement, c'est interdit. \\
\hline A3 & Pascal: & [8] Nous partirons demain. \\
\hline B3 & Mireille: & [9] D'accord. [10] Installez-vous dans le bois de pins. \\
\hline
\end{tabular}




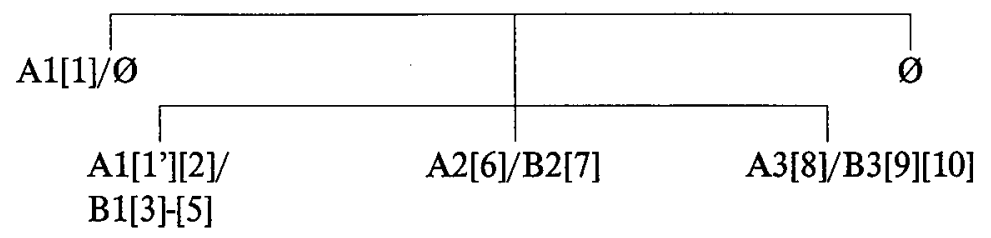

2.6 Analyse des textes du corpus selon le critère du type de texte

Les textes proposés dans les manuels et constituant notre corpus (les manuels sont cités dans l'introduction) ont été répartis en deux catégories:

- les textes qui introduisent les nouveaux thèmes de chaque dossier, écrits pour les manuels et donc inauthentiques,

- les autres textes (authentiques, authentiques adaptés et inauthentiques).

L'hypothèse de départ est la suivante: présence de tous les types de textes/séquences dans les manuels.

Dans la première catégorie de textes (textes pédagogiques) prédominent les séquences dialogales, les autres types de séquences sont très peu représentées.

L'analyse des textes de la deuxième catégorie, dans laquelle nous avons mis tous les autres textes, a donné les résultats suivants:

\begin{tabular}{|l|l|l|l|l|l|l|}
\hline & narrat. & $\begin{array}{l}\text { descript./ } \\
\text { listes }\end{array}$ & explicat. & argumentat. & dialogues & TOTAL \\
\hline NSF & 1 & $9 / 5$ & 1 & 1 & 1 & 18 \\
\hline NSF-c/ex & 7 & $9 / 11$ & 4 & 1 & $\emptyset$ & 32 \\
\hline P & 7 & $18 / 24$ & 9 & 1 & 4 & 63 \\
\hline P-c/ex & 5 & $11 / 4$ & 16 & 3 & 2 & 41 \\
\hline C & 9 & $28 / 16$ & 19 & $\emptyset$ & 2 & 74 \\
\hline C-c/ex & 1 & 2 & $\emptyset$ & 1 & $\emptyset$ & 4 \\
\hline
\end{tabular}

Cette catégorie de textes propose tous les types de séquences avec une nette prépondérance de narrations et de descriptions. Ces résultats ne surprennent pas vu que dans la vie de tous les jours les textes relevant de ces deux types sont sans doute les plus courants. Ce qui surprend est le fait que les séquences explicatives, argumentatives et dialogales sont très peu présentes. Le discours scolaire est l'exemple du type explicatif par excellence, l'explication est donc familière aux élèves et il est très facile de trouver des textes de ce type. Les textes argumentatifs n'apparaissent que plus tard dans l'apprentissage. Ce n'est pas très logique et c'est très dommage car l'enfant a très tôt besoin d'exprimer et de justifier ses points de vue. Le dialogue est le type de texte qui pourrait introduire un peu de dynamique dans les manuels: les entretiens (authentiques) avec les sportifs, célébrités, etc. pourraient être employés pour couvrir les thèmes traités, parfois même pour remplacer les textes pédagogiques introduisant les dossiers. 


\section{L'AUTHENTICITÉ DES TEXTES}

Cuq et Gruca (2002: 391) définissent les documents ${ }^{13}$ authentiques comme «des documents 'bruts', élaborés par des francophones pour des francophones à des fins de communication. Ce sont donc des énoncés produits dans des situations réelles de communication et non en vue de l'apprentissage d'une seconde langue». D'après l'enquête parmi les enseignants (Demari, 2004) et comme le note aussi Lewkowicz (1997: 166), il n'y a pas un vrai consensus quant à la définition de l'authenticité. Certains enseignants emploient en classe les documents tels quels, d'autres les raccourcissent, changent les mots jugés trop difficiles et considèrent ces textes modifiés comme étant encore authentiques. Dans le milieu des enseignants on emploie souvent l'expression textes semi-authentiques. La définition de cette expression n'est pas très claire: s'agit-il de textes authentiques adaptés ou de textes pédagogiques imitant les textes authentiques? ${ }^{14}$

Pour l'analyse des textes de notre corpus, nous avons réparti les textes dans les catégories suivantes:

- textes authentiques (A): textes écrits à d'autres fins, employés tels quels dans les manuels, avec la citation de la source;

- textes adaptés (Ad): textes adaptés d'après les textes authentiques, suivis de la citation «d'après»;

- textes qu'on qualifierait de prime abord authentiques, mais sans citation de la source (?A): horaires de trains, affiches, etc.;

- textes dont on ne peut pas déterminer l'authenticité, probablement des textes pédagogiques (?).

L'analyse de 200 textes a donné les résultats suivants:

\begin{tabular}{|l|l|l|l|l|l|}
\hline & Total & $?$ & $? \mathrm{~A}$ & Ad & A \\
\hline NSF & 28 & 24 & 3 & $\emptyset$ & 1 \\
\hline NSF-c/ex & 38 & 17 & 8 & $\emptyset$ & 13 \\
\hline P & 52 & 39 & 6 & 1 & 6 \\
\hline P-c/ex & 23 & 16 & 2 & $\emptyset$ & 5 \\
\hline C & 48 & 37 & 6 & 1 & 4 \\
\hline C-c/ex & 11 & 7 & 3 & $\emptyset$ & 1 \\
\hline
\end{tabular}

Les résultats de l'analyse sont surprenants: on peut voir qu'il y a dans les manuels un nombre très limité de textes authentiques et que le plus grand nombre

13 II ne s'agit pas seulement de textes, mais aussi de photos, vidéos, etc.

14 Dans l'enquête, réalisée auprès de 15 enseignants slovènes, la majorité d'entre eux affirment utiliser des documents authentiques. Pourtant certains citent comme unique source certains journaux de Mary Glasgow Magazines qui, à notre connaissance, n'emploient que des textes inauthentiques. 
de textes authentiques apparaît dans la méthode la plus ancienne (NSF: 14 en tout, contre 11 dans Panorama et seulement 5 dans la méthode la plus récente, Campus).

Il n'y a pratiquement pas de textes adaptés (1 dans Panorama et 1 dans Campus).

Parmi les textes qu'on qualifierait de prime abord authentiques on peut trouver les horaires des trains, les programmes des spectacles, différents formulaires, etc. Malheureusement les auteurs les emploient sans citation de source.

Dans les trois manuels on note une nette prépondérance des textes inauthentiques, probablement écrits pour les méthodes.

La question qui s'impose après cette analyse est la suivante: pourquoi il y a-t-il moins de textes authentiques dans le manuel le plus récent et surtout pourquoi les auteurs des manuels n'emploient-ils pas plus de textes adaptés qui constitueraient en fait un bon substitut des textes authentiques.

\section{EXPERIMENTATION EN CLASSE}

Avec l'expérimentation en classe nous avons essayé de voir quelle est l'influence du type et de l'authenticité du texte sur la compréhension. Pour la formulation des hypothèses nous nous sommes appuyés sur les résultats de l'analyse du corpus.

L'expérimentation a été menée en $3^{\mathrm{e}}$ (111 élèves de $17 / 18$ ans), en $4^{\mathrm{e}}$ ( 66 élèves de 18/19 ans) année de lycée et en lère année de faculté (17 étudiants de 19 à 22 ans).

Les exercices ont été élaborés après consultation préalable avec les professeurs ${ }^{15}$ du secondaire et prétestés deux fois (sur 18 et 27 élèves) ${ }^{16}$. Nous avons été limités par le nombre d'élèves qui apprennent le français en Slovénie: ce nombre étant relativement petit, nous n'avons pas pu nous permettre un échantillon plus important. Les hypothèses sont les suivantes:

- les lycéens et les étudiants ont plus de facilité à reconnaître certains types de textes; notamment ceux auxquels ils sont - selon les résultats de l'analyse du corpus - plus souvent exposés;

- l'identification du type de texte les aide à mieux résoudre les exercices;

- les textes adaptés sont plus faciles et donc mieux compris.

Pour l'expérimentation nous avons élaboré un ensemble d'exercices avec différents types de textes, proposant à chaque fois une variante authentique et une variante adaptée $^{17}$ :

\footnotetext{
15 Nous leur avons demandé leur avis sur le choix des textes et sur les adaptations proposées.

16 Nous avons inclus les élèves des lycées Be igrad (Ljubljana), Kranj, Škofja Loka, Poljane (Ljubljana) et Šentvid (Ljubljana).

17 Adaptation de la structure, remplacement des termes jugés trop difficiles par des termes plus courants, omission des informations qui ne sont pas importantes, ajout $d$ ' informations qui pourraient faciliter la compréhension, explication des termes liés à la civilisation.
} 
- texte à dominante narrative: questions orientées;

- texte à dominante descriptive: questions fermées (vrai/faux);

- texte à dominante explicative: exercice lacunaire;

- texte à dominante argumentative: exercice à choix multiple;

- texte à dominante argumentative (après avoir été prétesté jugé trop difficile pour les lycéens, donc testé auprès d'un certain nombre d'étudiants de la Faculté des lettres): questions orientées.

Dans le choix des types d'exercices nous avons été limités par le temps qui nous était attribué pour tester chacune des classes (1 leçon, c'est-à-dire 45 minutes). Les questionnaires à réponses ouvertes (que nous avons employés lors du premier pré-test) nous auraient donné plus d'informations sur la compréhension, malheureusement il nous a été impossible de les employer ${ }^{r}$, faute de temps.

Les résultats de l'expérimentation ${ }^{18}$ montrent une meilleure identification des textes à dominante narrative et descriptive, ce qui n'a rien de surprenant, vu le nombre de textes de ces deux types dans les manuels.

Contrairement aux attentes, l'identification du type de texte n'a pas joué un rôle significatif dans la compréhension des textes.

Les exercices avec les textes adaptés n'ont pas révélé une meilleure compréhension que les exercices avec les textes authentiques. Une meilleure compréhension du texte adapté n'a été relevée que chez les étudiants - où nous avons testé le texte argumentatif, trop difficile pour les lycéens - mais vu leur petit nombre (17) ce résultat ne peut pas être statistiquement significatif.

18 Pour les résultats détaillés, voir la thèse de doctorat intitulée Vpliv tipa in avtentičnosti besedila na njegovo razumevanje. 


\section{PERSPECTIVES}

A partir de nos analyses on peut constater qu'une meilleure sensibilisation à la typologie textuelle en classe de FLE serait très utile: outre les narrations et les descriptions, il faudrait habituer les élèves - lecteurs aux autres types de textes, surtout aux explications et aux argumentations. Le fait de pouvoir reconnaître et maîtriser différents types de textes ne les aiderait pas seulement dans le domaine de la compréhension écrite, mais aussi dans celui de l'expression, aussi bien orale qu' écrite.

Selon les résultats de notre recherche, l'adaptation des textes pour la compréhension écrite n'est pas nécessaire. Nous serions cependant plus prudents quant à l'emploi de textes authentiques lors de l'évaluation: quand l'élève est sanctionné par une note, une adaptation des textes semble toujours raisonnable, même si c'est seulement pour sécuriser l'élève (et son professeur). En classe pourtant il serait préférable d'utiliser des textes authentiques afin d'ancrer les apprenants au mieux dans la réalité francophone et dans la vie de tous les jours. 


\section{BIBLIOGRAPHIE}

ADAM, J.-M. (1985): Quels types de textes, in Le français dans le monde, $\mathrm{n}^{\circ} 192$, pp. 39-43.

ADAM, J.-M. (1991): Cadre théorique d'une typologie séquentielle, in: Études de linguistique appliquée n ${ }^{\circ}$ 83, pp. 7-18. ADAM, J.-M. (1994): Le texte narratif. Paris: Nathan.

ADAM, J.-M. (1997): Les textes: types et prototypes. Paris: Nathan Université.

ADAM, J.-M. (1999): Linguistique textuelle. Des genres de discours aux textes. Paris: Nathan Université.

ADAM, J.-M. (2001): Types de textes ou genres de discours? Comment classer les textes qui disent de et comment faire? in: Langages no $141, \mathrm{pp} .10-27$.

ADAM, J.-M., PETITJEAN, A. (1989): Le texte descriptif. Paris: Nathan.

BEŠTER, M., et al. (1999): Na pragu besedila: učbenik za slovenski jezikv 1 . letniku gimnazij, strokovnih in tehniških šol. Ljubljana: Rokus.

BOISSINOT, A. (1999): Les textes argumentatifs. Toulouse: Bertrand Lacoste CRDP Midi-Pyrénées.

CUQ, J.-P., GRUCA, I. (2002): Cours de didactique du français langue étrangère et seconde. Grenoble: Presses Universitaires de Grenoble.

DEMARI, J.-C. (2004): «Authentique», mode d'emploi, in: Le français dans le monde, $\mathrm{n}^{\circ} 331$, pp. 32-33.

ECO, U. (1979, traduction fr. 1985): Lector in fabula. Paris: Grasset.

JEANDILLOU, J.-F. (1997): LAnalyse textuelle. Paris: Armand Colin.

KINTSCH, W., VAN DIJK, T. A. (1978): Toward a Model of Text Comprehension and Production, in Psychological Review, vol. 85, $\mathrm{n}^{\circ}$ 5, pp. 363-394.

LAH, M. (2004): Vpliv tipa in avtentičnosti besedila na njegovo razumevanje. Ljubljana: Univerza v Ljubljani, Filozofska fakulteta.

LEWKOWICZ, J. A. (1997): Authentic for whom? Does authenticity really matter? in: Huhta, A. et al: Current Developments and Alternatives in Language Assessment - Proceedings of LTRC 96. Jyväskyla: University of Jyväskyla, pp. 164-184.

MOESCHLER, J. (1985): Argumentation et conversation. Paris: Hatier-Crédif.

POULIOT, M. (1993): Discours explicatif écrit en milieu universitaire, in: Le français dans le monde/Recherches et applications: Des pratiques de l'écrit, pp. 120-128.

REBOUL, A., MOESCHLER, J. (1998): Pragmatique du discours. Paris: Armand Colin.

ROULET, E. (1991): Une approche discursive de l'hétérogénéité discursive, in: Études de linguistique appliquée ${ }^{\circ}$ 83, pp. 117-130.

TAGLIANTE, C. (1994): La classe de langue. Paris: CLE International.

VIGNER, G. (1990): Un type de texte: le dire de faire, in: Pratiques n 66, pp. 107- 124.

Manuels

DOMINIQUE, P., GIRARDET, J., VERDELHAN, M. et M. (1998): Le nouveau sans frontières 1. Paris: CLE International.

VERDELHAN M. in M., DOMINIQUE, P. GIRARDET, J. (1988): Le nouveau sans frontières 1 , cahier d'exercices. Paris: CLE International.

GIRARDET, J. (1988): Le nouveau sans frontières 1, Le livre du professeur. Paris: CLE International.

GIRARDET, J., CRIDLIG, J.-M. (2000): Panorama. Paris: CLE International.

GIRARDET, J., CRIDLIG, J.-M. (1996): Panorama 1 - cahier d'exercices. Paris: CLE International.

GIRARDET, J., CRIDLIG, J.-M. (1996): Panorama 1 - livre du professeur. Paris: CLE International.

GIRARDET, J., PECHEUR, J. (2002): Campus. Paris: CLE International.

GIRARDET, J., PECHEUR, J. (2002): Campus. Cahier d'exercices. Paris: CLE International.

GIRARDET, J., PECHEUR, J. (2002): Campus. Livre du professeur. Paris: CLE International. 


\section{VPLIV TIPA IN AVTENTIČNOSTI BESEDILA NA NJEGOVO RAZUMEVANJE}

$\breve{C l a n e k ~ j e ~ p o v z e t e k ~ d o k t o r s k e ~ d i s e r t a c i j e ~ V p l i v ~ t i p a ~ i n ~ a v t e n t i c ̌ n o s t i ~ b e s e d i l a ~ n a ~ n j e g o v o ~ r a z u m e-~}$ vanje, ki jo je avtorica zagovarjala v juniju 2004 na Filozofski fakulteti Univerze v Ljubljani pred komisijo: doc. dr. Mojca Schlamberger Brezar (mentorica), red. prof. dr. Vladimir Pogačnik in izr. prof. dr. Ana Marija Muster.

Namen disertacije je bil bolje raziskati vlogo, ki jo pri razumevanju igra sámo besedilo, predvsem dva od njegovih aspektov, tip in avtentičnost.

Avtorica je analizo tipa in avtentičnosti besedil opravila $v$ treh učbeniških kompletih (Le nouveau sans frontières, Panorama in Campus), ki jih je izbrala zaradi njihove razširjenosti v Sloveniji (prva dva kompleta) in prisotnosti iste avtorice pri vseh treh (J. Girardet). Za analizo je uporabila Adamovo tipologijo, ki jo predstavlja v prvem delu članka. Adam besedila deli na pripovedi, opise, razlage, utemeljevanja in dialoge. Kategorijo dialoga je, kljub temu, da je nekoliko sporna, avtorica ohranila za analizo uvodnih besedil v lekcije. Ugotavlja, da so uvodna besedila v glavnem dialoška, med ostalimi besedili v učbenikih pa v pretežni meri zasledimo pripovedi in opise. Razlage, utemeljevanja in dialogi so $\mathrm{v}$ učbenikih le redko prisotni.

$\mathrm{Za}$ analizo avtentičnosti besedil se je odločila, ker se besedila za poučevanje in preverjanje razumevanja pogosto prirejajo, ni pa povsem jasno, kakšno vlogo ima prirejanje besedil pri razumevanju. Za analizo besedil v učbenikih je beseđila razdelila na več kategorij: avtentična, prirejena z navedbo vira, navidezno avtentična in besedila, ki jim avtentičnosti ne moremo določiti, so pa po vsej verjetnosti pedagoška. $V$ učbenikih je zdaleč največ besedil, ki jim avtentičnosti ne moremo določiti. C̆udi majhno število avtentičnih besedil (ki je največje v najstarejšem učbeniku, Le nouveau sans frontières) in še manjše število prirejenih besedil.

$\checkmark$ eksperimentalnem delu je avtorica izvedla poskus s 177 dijaki 3. in 4. letnikov gimnazij in 17 študenti 1. letnika Filozofske fakultete v Ljubljani. Poskus je izvedla $\mathrm{z}$ avtentičnimi in prirejenimi besedili različnih tipov. Glede na rezultate analize korpusa je predvidevala, da bodo dijaki bolje prepoznavali pripovedi in opise, kar se je potrdilo. Prepoznavanje tipa besedila ni vplivalo na boljše reševanje nalog, prav tako se naloge, pripravljene $z$ avtentičnimi besedili, niso izkazale za težje od tistih s prirejenimi.

Sklep raziskave je, da bi morali dijake bolj navajati na prepoznavanje in uporabo različnih tipov besedil (zlasti tistih, ki so v učbenikih manj prisotni, npr. razlaga in utemeljevanje) in v razredu uporabljati predvsem avtentična besedila. Za preverjanje in ocenjevanje pa se zdi uporaba prirejenih besedil še vedno smiselna. 